\title{
Non-contiguous finished genome sequence and description of Collinsella massiliensis sp. nov.
}

\author{
Roshan Padmanabhan ${ }^{1+}$, Gregory Dubourg ${ }^{1+}$, Jean-Christophe lagier ${ }^{1}$, Thi-Thien Ngu- \\ yen $^{1}$, Carine Couderc ${ }^{1}$, Morgane Rossi-Tamisier ${ }^{1}$, Aurelia Caputo ${ }^{1}$, Didier Raoult ${ }^{1,2}$ and \\ Pierre-Edouard Fournier ${ }^{1 *}$ \\ ${ }^{1}$ Unité de Recherche sur les Maladies Infectieuses et Tropicales Emergentes, Institut \\ Hospitalo-Universitaire Méditerranée-Infection, Faculté de médecine, Aix-Marseille \\ Université, Marseille cedex 05, France \\ ${ }^{2}$ King Fahd Medical Research Center, King Abdulaziz University, Jeddah, Saudi Arabia \\ *Correspondence: Pierre-Edouard Fournier (pierre-edouard.fournier@univ-amu.fr) \\ $\dagger$ Both authors participated equally to this work
}

Key words: Collinsella massiliensis, genome, culturomics, taxono-genomics

\begin{abstract}
Collinsella massiliensis strain $\mathrm{GD}^{\top}$ is the type strain of Collinsella massiliensis sp. nov., a new species within the genus Collinsella. This strain, whose genome is described here, was isolated from the fecal flora of a 53-year-old French Caucasoid woman who had been admitted to intensive care unit for Guillain-Barré syndrome. Collinsella massiliensis is a Gram-positive, obligate anaerobic, non motile and non sporulating bacillus. Here, we describe the features of this organism, together with the complete genome sequence and annotation. The genome is 2,319,586 bp long ( 1 chromosome, no plasmid), exhibits a $\mathrm{G}+\mathrm{C}$ content of $65.8 \%$ and contains 2,003 protein-coding and 54 RNA genes, including 1 rRNA operon.
\end{abstract}

\section{Introduction}

Collinsella massiliensis strain GD3 ${ }^{\mathrm{T}}$ (= CSUR P902 = DSM 26110) is the type strain of $C$. massiliensis sp. nov. This bacterial strain was isolated from the fecal flora of a 53-year-old French Caucasoid female admitted to the intensive care unit (ICU) in the Timone Hospital of Marseille, France, for Guillain-Barré syndrome. This study was part of a "culturomics" effort to cultivate all bacteria within human feces [1]. C. massiliensis is a Grampositive, obligatly anaerobic, non-endospore forming, non-motile and rod shaped bacillus.

Thanks to the development of high throughput sequencers and the rapidly declining cost of genome sequencing, the number of sequenced bacterial genomes has reached almost 12,000 as of January $2^{\text {nd }}, 2014$, with an additional 18,000 sequencing projects ongoing [2]). In an effort to include genomic information among the genotypic criteria used for the taxonomic description of bacterial isolates, and not only rely on a combination of $16 \mathrm{~S}$ rRNA gene phylogeny and nucleotide sequence similarity, $\mathrm{G}+\mathrm{C}$ content and DNA-DNA hybridization [3-6]. We proposed a new strategy named taxono-genomics that we used to describe several new bacterial taxa [738].
In 1999, Kageyama et al. reclassified Eubacterium aerofaciens into a new genus named Collinsella [39] based on a 16S rRNA gene sequence divergence and the presence of a unique peptidoglycan type when compared to other members of the genus Eubacterium. In addition to the type species, C. aerofaciens [39], the genus Collinsella currently includes $C$. intestinalis [40], C. stercoris [40] and C. tanakaei [41]. All four species have been isolated from the human gastrointestinal tract.

In the present manuscript, we apply the taxonogenomics strategy to the description of Collinsella massiliensis sp. nov., and describe the complete genome sequencing and annotation of Collinsella massiliensis strain GD3' (= CSUR P902 = DSM 26110). These characteristics support the circumscription of the $C$. massiliensis species.

\section{Classification and Features}

A stool sample was collected from a 53-year-old female admitted to the intensive care unit of the Timone Hospital in Marseille, France, for Guillain-Barré syndrome. The patient gave a written informed consent for the study, which was approved by the Ethics Committee of the 
Padmanabhan et al.

Institut Fédératif de Recherche 48, Faculty of Medicine, Marseille, France, under agreement number 09-022. She received antibiotics at the time of stool sample collection and the fecal specimen was preserved at $-80^{\circ} \mathrm{C}$ immediately after collection. Strain GD3 ${ }^{\mathrm{T}}$ (Table 1) was first isolated in January 2012 after incubation for two weeks in an anaerobic blood culture bottle that also contained clarified and sterile sheep rumen. Then, the strain was sub-cultivated anaerobically at $37^{\circ} \mathrm{C}$ on $5 \%$ sheep blood-enriched Columbia agar (BioMerieux, Marcy l'Etoile, France). Several other new bacterial species were isolated from this stool specimen using various culture conditions.
When compared to sequences available in GenBank, the 16s rRNA sequence of $C$. massiliensis strain GD3 ${ }^{\mathrm{T}}$ (GenBank accession number JX424766) exhibited the highest sequence identity of $95.7 \%$ with Collinsella tanakaei (Figure 1). This value was lower than the threshold (98.7\%) recommended by Stackebrandt and Ebers to delineate a new species without carrying out DNA-DNA hybridization [4], and was in the range of 16S rRNA identity values observed among the four Collinsella species with validly published names $(92.2$ between C. intestinalis and C. aerofaciens to $97.7 \%$ between C. intestinalis and C. stercoris) [49].

Table 1. Classification and general features of Collinsella massiliensis strain GD3 ${ }^{\top}$ according to the MIGS recommendations [42].

\begin{tabular}{|c|c|c|c|}
\hline MIGS ID & Property & Term & Evidence code $^{a}$ \\
\hline & Current classification & Domain Bacteria & TAS [43] \\
\hline & & Phylum Actinobacteria & TAS [44] \\
\hline & & Class Actinobacteria & TAS [45] \\
\hline & & Order Coriobacteriales & TAS $[45,46]$ \\
\hline & & Family Coriobacteriaceae & TAS [47] \\
\hline & & Genus Collinsella & TAS [39] \\
\hline & & Species massiliensis & IDA \\
\hline & & Type strain GD $3^{\top}$ & IDA \\
\hline & Gram stain & Positive & IDA \\
\hline & Cell shape & Bacilli & IDA \\
\hline & Motility & Non motile & IDA \\
\hline & Sporulation & Non spore forming & IDA \\
\hline & Temperature range & Mesophilic & IDA \\
\hline & Optimum temperature & $37^{\circ} \mathrm{C}$ & IDA \\
\hline MIGS-6.3 & Salinity & Unknown & NAS \\
\hline \multirow[t]{3}{*}{ MIGS-22 } & Oxygen requirement & Anaerobic & IDA \\
\hline & Carbon source & Unknown & NAS \\
\hline & Energy source & Unknown & NAS \\
\hline MIGS-6 & Habitat & Human gut & IDA \\
\hline MIGS-15 & Biotic relationship & Free living & IDA \\
\hline \multirow[t]{3}{*}{ MIGS-14 } & Pathogenicity & Unknown & IDA \\
\hline & Biosafety level & 2 & \\
\hline & Isolation & Human feces & \\
\hline MIGS-4 & Geographic location & France & IDA \\
\hline MIGS-5 & Sample collection time & January 2012 & IDA \\
\hline MIGS-4.1 & Latitude & 43.296482 & IDA \\
\hline MIGS-4.1 & Longitude & 5.36978 & IDA \\
\hline MIGS-4.3 & Depth & Surface & IDA \\
\hline MIGS-4.4 & Altitude & $0 \mathrm{~m}$ above sea level & IDA \\
\hline
\end{tabular}

Evidence codes - IDA: Inferred from Direct Assay; TAS: Traceable Author Statement (i.e., a direct report exists in the literature); NAS: Non-traceable Author Statement (i.e., not directly observed for the living, isolated sample, but based on a generally accepted property for the species, or anecdotal evidence). These evidence codes are from the Gene Ontology project [48]. If the evidence is IDA, then the property was directly observed for a live isolate by one of the authors or an expert mentioned in the acknowledgements. 


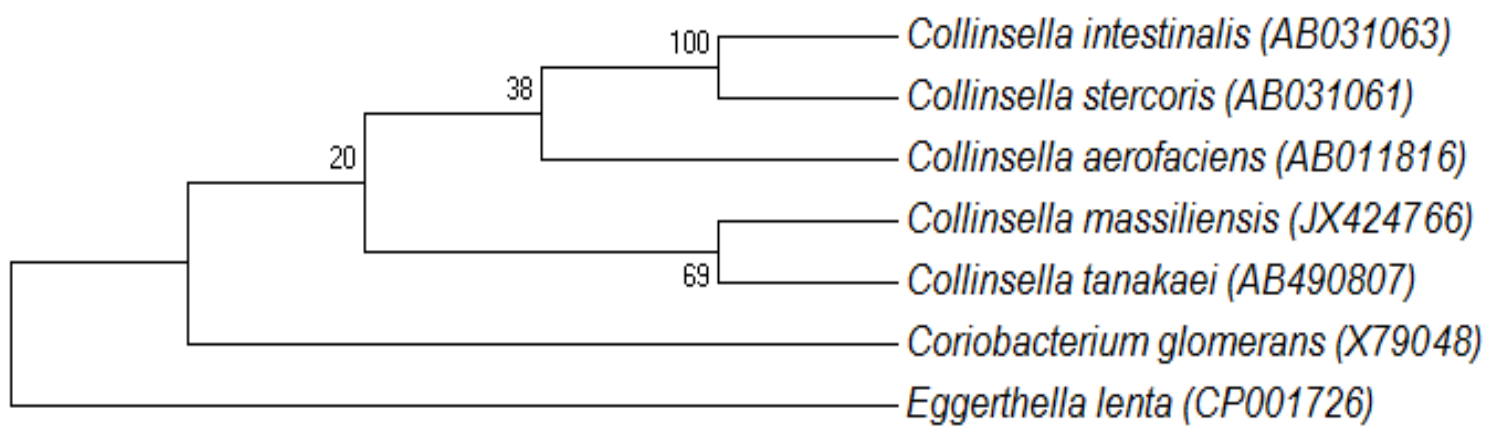

Figure 1: A consensus phylogenetic tree highlighting the position of Collinsella massiliensis strain $\mathrm{GD}^{\top}$ relative to other type strains within the genus Collinsella. GenBank accession numbers are indicated in parentheses. Sequences were aligned using CLUSTALW and phylogenetic inferences obtained using the neighbor-joining method within the MEGA software. Numbers at the nodes are percentages of bootstrap values obtained analysis from1,000 REPLICTES to generate a majority consensus tree. Eggerthella lenta was used as an outgroup.

Growth of the strain was tested in 5\% sheep blood-enriched Columbia agar (BioMerieux) under anaerobic and microaerophilic conditions (GENbag anaer and GENbag microaer systems, respectively, BioMerieux), and in aerobic conditions, with or without $5 \% \mathrm{CO}_{2}$. Growth was achieved only anaerobically. In addition, among the four different incubation temperatures tested $\left(25,30,37,45^{\circ} \mathrm{C}\right)$, no growth was observed at $25^{\circ} \mathrm{C}$ and $30^{\circ} \mathrm{C}$ but strain GD3 ${ }^{\mathrm{T}}$ grew at 37 and $45^{\circ} \mathrm{C}$. The best growth was obtained at $37^{\circ} \mathrm{C}$ after 48 hours of incubation. Colonies were grey, translucent and $0.4 \mathrm{~mm}$ in diameter on bloodenriched Columbia agar. Gram staining showed Gram-positive rods unable to form spores (Figure 2). A motility test was negative. In electron microscopy, cells grown on agar had a mean diameter of $0.57 \mu \mathrm{m}$, a mean length of $1.19 \mu \mathrm{m}$ (Figure 3) and were mostly grouped in short chains or small clumps.

Strain GD3 ${ }^{\mathrm{T}}$ showed neither catalase nor oxidase activities. Using an API ZYM strip (BioMerieux), positive reactions were observed for acid phosphatase, naphthol-AS-BI-phosphohydrolase, $\alpha$ galactosidase, alkaline phosphatase, leucine arylamidase, $\alpha$-glucosidase. Negative reactions were observed for cystin arylamidase, $\beta$ glucuronidase, nitrate reduction, urease, esterase (C4), esterase lipase (C8), lipase (C14), Trypsin, $\alpha$-chemotrypsin, $\quad \mathrm{N}$-actetyl- $\beta$-glucosaminidase, $\alpha$-mannosidase and $\alpha$-fucosidase. Using an API Rapid ID 32A strip (BioMerieux), positive reactions were observed for $\alpha$-galactosidase, $\alpha$ glucosidase, $\alpha$-fucosidase, leucine arylamidase, proline arylamidase, arginine dihydrolase, serine arylamidase and glycine arylamidase. Negative reactions were observed for histidin arylamidase, urease, phenylalanine arylamidase, tyrosin arylamidase, leucyl-glycyl arylamidase, alanine arylamidase, and arginine arylamidase. Using an API $50 \mathrm{CH}$ strip (BioMerieux), positive reactions were obtained for D-sorbitol, Dsaccharose, xylitol, D-arabitol and potassium-5ketogluconate. Negative reactions were observed for the fermentation of glycerol, erythritol, D-arabinose, L-arabinose, D-ribose, Dxylose, L-xylose, D-adonitol, methyl- $\beta$-Dxylopranoside, D-galactose, D-glucose, Dfructose, D-mannose, L-sorbose, L-rhamnose, dulcitol, inositol, D-mannitol, methyl- $\alpha \mathrm{D}$ xylopranoside, methyl- $\alpha \mathrm{D}$-glucopranoside, Nacetylglucosamine, amygdalin, arbutin, esculin ferric citrate, salicin, D-cellobiose, D-maltose, Dlactose, D-mellibiose, D-trehalose, inulin, Dmelezitose, D-raffinose, amidon, glycogen, gentiobiose, D-turanose, D-lyxose, D-tagatose, Lfucose, L-arabitol, potassium gluconate and potassium 2-ketogluconate. Collinsella massiliensis is susceptible to penicillin $\mathrm{G}$, amoxicillin, amoxicillin-clavulanic acid, ceftriaxon, imipenem, metronidazole, vancomycin, rifampicin but resistant to erythromycin, gentamicin, ciprofloxacin and trimethoprim/sulfa-methoxazole. By comparison with all other Collinsella species (Table 2), C. massiliensis differed in production of arginine arylamidase, leucine arylamidase, leucyl-glycyl arylamidase, and acidification of glucose, mannose, galactose, fructose and sorbitol. 


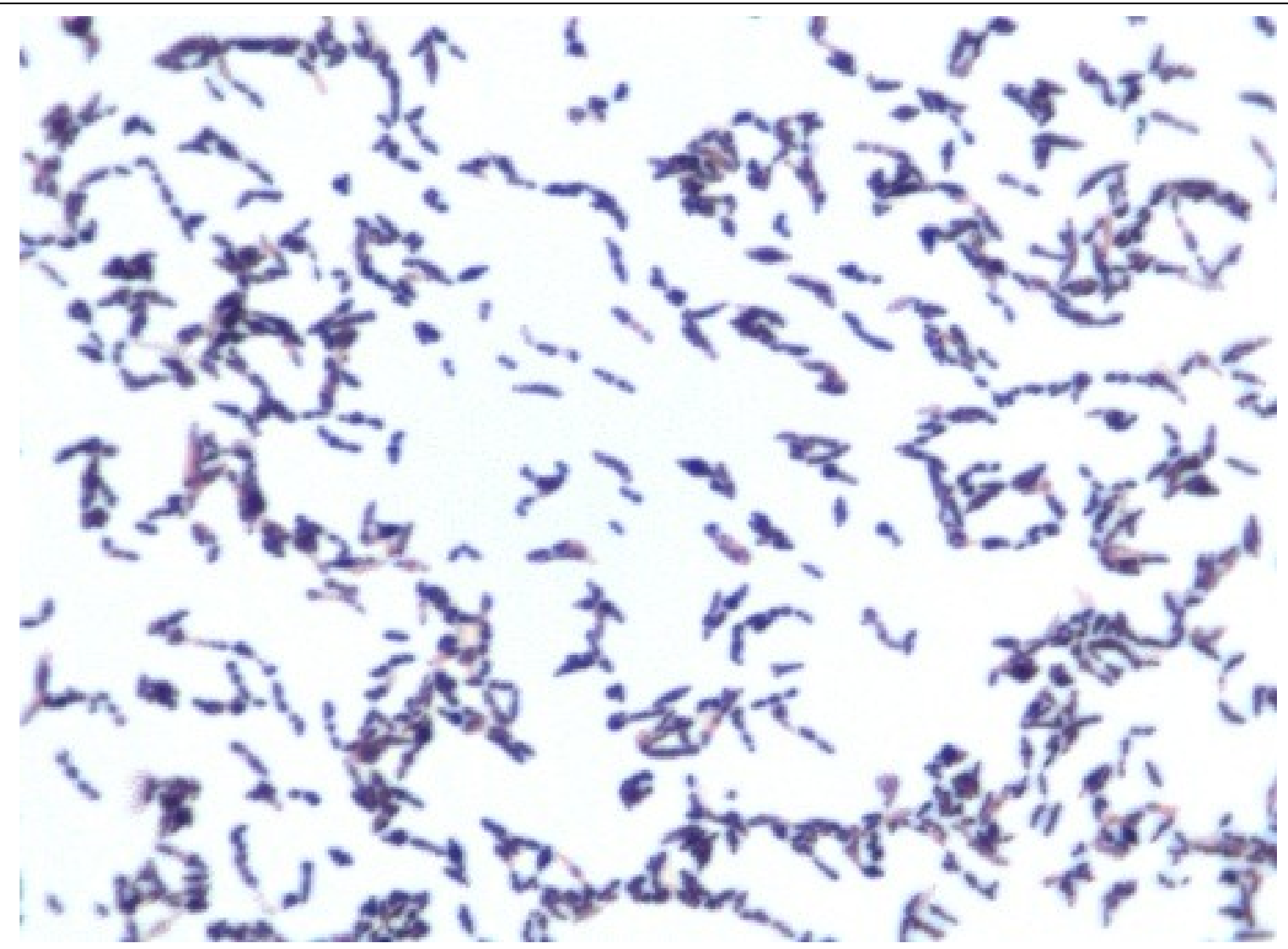

Figure 2: Gram staining of Collinsella massiliensis strain $\mathrm{GD}^{\top}$

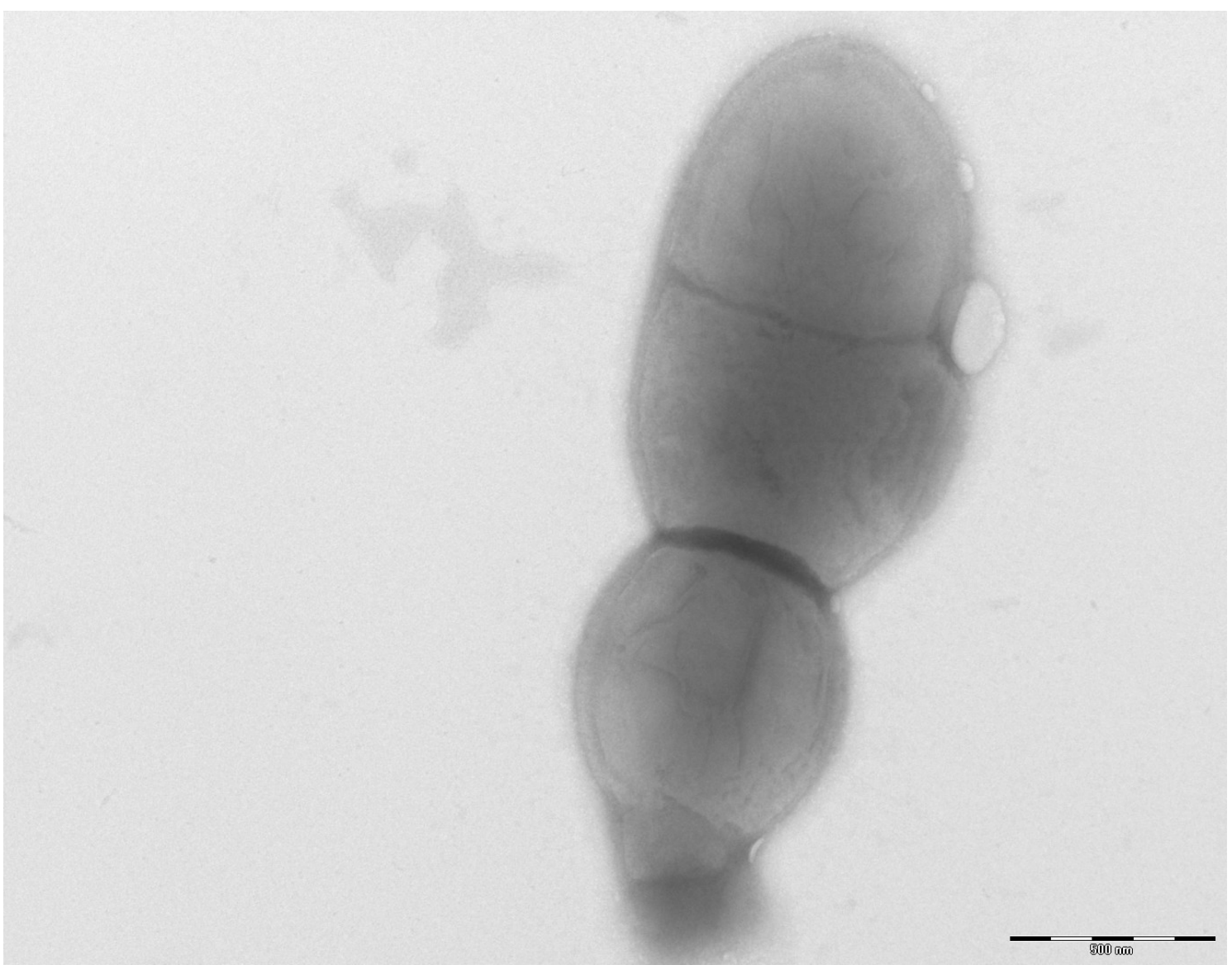

Figure 3: Transmission electron microscopy of Collinsella massiliensis strain GD3 ${ }^{\top}$, made using a Morgagni 268D (FEI Electron Optics, Hillsboro, OR, USA) at an operating voltage of $60 \mathrm{kV}$. The scale bar represents $500 \mu \mathrm{m}$. 
Table 2: Differential characteristics of Collinsella massiliensis strain GD3 ${ }^{\top}$ with other strains

\begin{tabular}{|c|c|c|c|c|c|}
\hline Properties & C. massiliensis & C. intestinalis & $\begin{array}{c}C . \\
\text { aerofaciens }\end{array}$ & C. tanakei & C. stercoris \\
\hline Cell diameter $(\mu \mathrm{m})$ & 0.57 & $0.3-0.5$ & $0.3-0.7$ & $0.5-1.0$ & $0.3-0.5$ \\
\hline Oxygen requirement & anaerobic & anaerobic & anaerobic & anaerobic & anaerobic \\
\hline Gram stain & + & + & + & + & + \\
\hline Motility & - & - & na & - & - \\
\hline Endospore formation & - & - & - & na & - \\
\hline \multicolumn{6}{|l|}{ Production of } \\
\hline Alkaline phosphatase & + & + & - & + & + \\
\hline Acid phosphatase & + & + & - & + & + \\
\hline Catalase & - & na & na & - & na \\
\hline Oxidase & - & na & na & - & na \\
\hline Nitrate reductase & - & na & na & - & na \\
\hline Urease & - & na & - & - & na \\
\hline$\alpha$-galactosidase & + & - & + & - & - \\
\hline$\beta$-galactosidase & + & - & + & - & + \\
\hline$\beta$-glucuronidase & - & - & - & + & - \\
\hline$\alpha$-glucosidase & + & - & + & - & - \\
\hline$\beta$-glucosidase & - & - & - & + & + \\
\hline Esterase & - & na & - & - & na \\
\hline Esterase lipase & - & - & - & - & - \\
\hline Indole & - & na & na & - & na \\
\hline $\begin{array}{l}\mathrm{N} \text {-acetyl- } \beta \text { - } \\
\text { glucosaminidase }\end{array}$ & - & + & - & - & + \\
\hline Alanine arylamidase & - & na & - & - & + \\
\hline Arginine arylamidase & - & na & + & + & na \\
\hline Cystin arylamidase & - & - & - & - & - \\
\hline $\begin{array}{l}\text { Glutamic acid decar- } \\
\text { boxylase }\end{array}$ & - & na & - & - & na \\
\hline Glycine arylamidase & + & na & + & + & na \\
\hline Leucine arylamidase & - & + & + & + & + \\
\hline $\begin{array}{l}\text { Leucyl-glycyl } \\
\text { arylamidase }\end{array}$ & - & na & + & + & na \\
\hline Proline arylamidase & + & - & + & + & - \\
\hline Serine arylamidase & + & na & - & - & + \\
\hline Tyrosin arylamidase & - & na & - & - & + \\
\hline Valine arylamidase & - & - & + & - & - \\
\hline \multicolumn{6}{|l|}{ Utilization of } \\
\hline Glucose & - & + & + & + & + \\
\hline Mannose & - & + & + & + & + \\
\hline Galactose & - & + & + & na & + \\
\hline Fructose & - & + & + & na & + \\
\hline Maltose & - & - & + & + & + \\
\hline Cellobiose & - & - & - & + & + \\
\hline Lactose & - & - & + & + & + \\
\hline L-arabinose & - & - & - & - & - \\
\hline D-xylose & - & - & - & - & - \\
\hline Rhamnose & - & - & na & - & - \\
\hline Ribose & - & - & + & + & + \\
\hline Raffinose & - & - & na & na & - \\
\hline Glycogen & - & - & na & na & - \\
\hline Aesculin & - & - & - & - & - \\
\hline Mannitol & - & - & - & na & - \\
\hline Sorbitol & + & - & - & - & - \\
\hline Habitat & human gut & human gut & human gut & human gut & na \\
\hline
\end{tabular}

na: data not available; +/-: depending on tests used 
Padmanabhan et al.

Matrix-assisted laser-desorption/ionization time-of-flight (MALDI-TOF) MS protein analysis was peformed as previously described [50] using a Microflex spectrometer (Bruker Daltonics, Leipzig, Germany). The spectra from 12 distinct colonies from a culture agar plate were imported into the MALDI BioTyper software (version 2.0, Bruker) and analyzed by standard pattern matching (with default parameter settings) against the main spectra of 4,706 bacteria including 2 spectra from Collinsella aerofaciens, that were part of the reference data contained in the BioTyper database. The resulting score enabled the presumptive identification and discrim- ination of the tested isolate from those in the database according to the following rule: a score $>$ 2 with a validated species enabled the identification at the species level; a score $>1.7$ but $<2$ enabled the identification at the genus level; and a score $<1.7$ did not enable any identification. No significant score was obtained for strain GD3T, suggesting that the isolate was not a member of any known species. The reference mass spectrum of Collinsella massiliensis strain $\mathrm{GD}^{\mathrm{T}}$ and the gel view comparing this spectrum with other phylogenetically close species are presented in Figures 4 and 5, respectively.

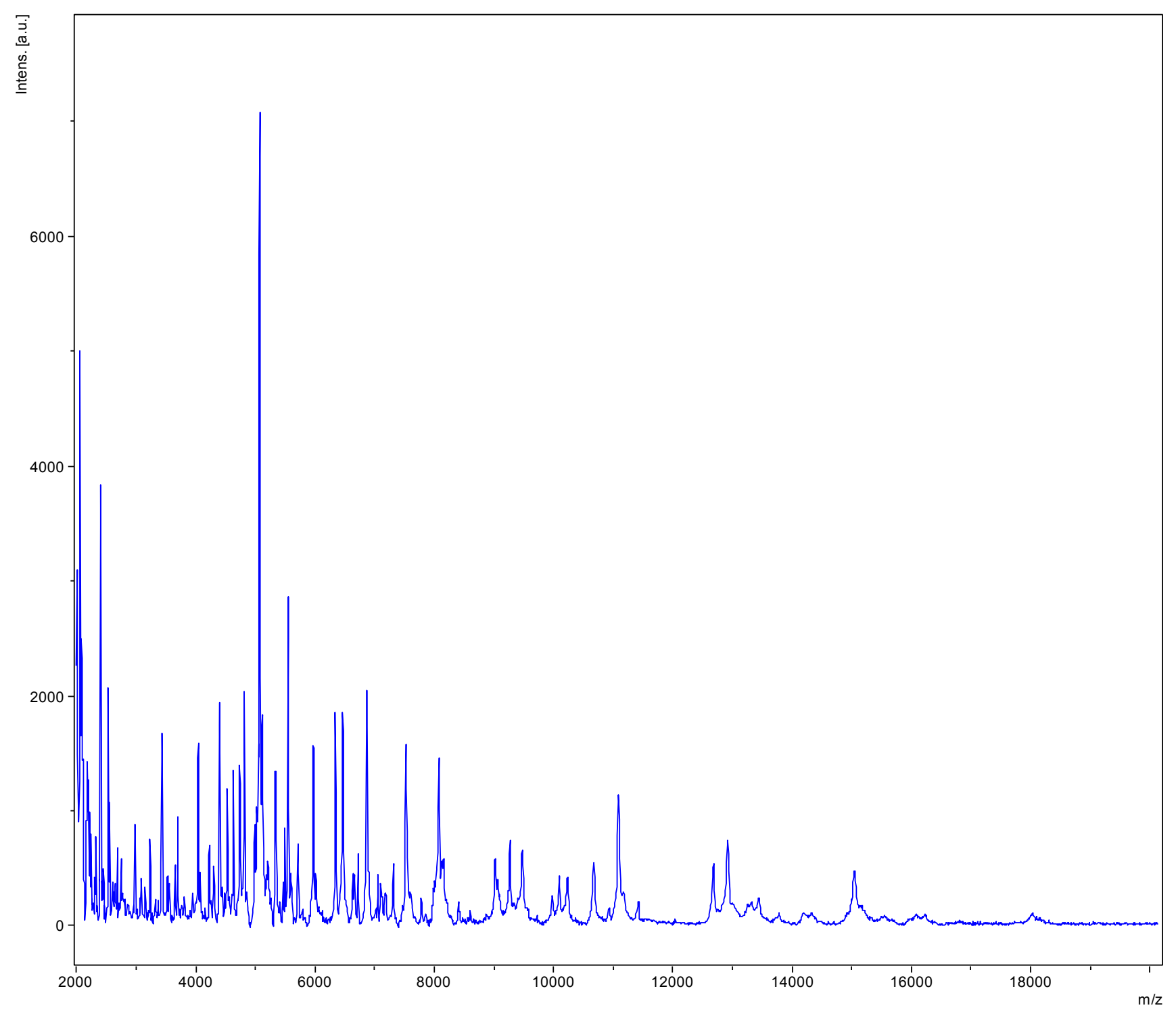

Figure 4: Reference mass spectrum from Collinsella massiliensis strain GD ${ }^{\top}$. Spectra from 12 individual colonies were compared and a reference spectrum was generated. 


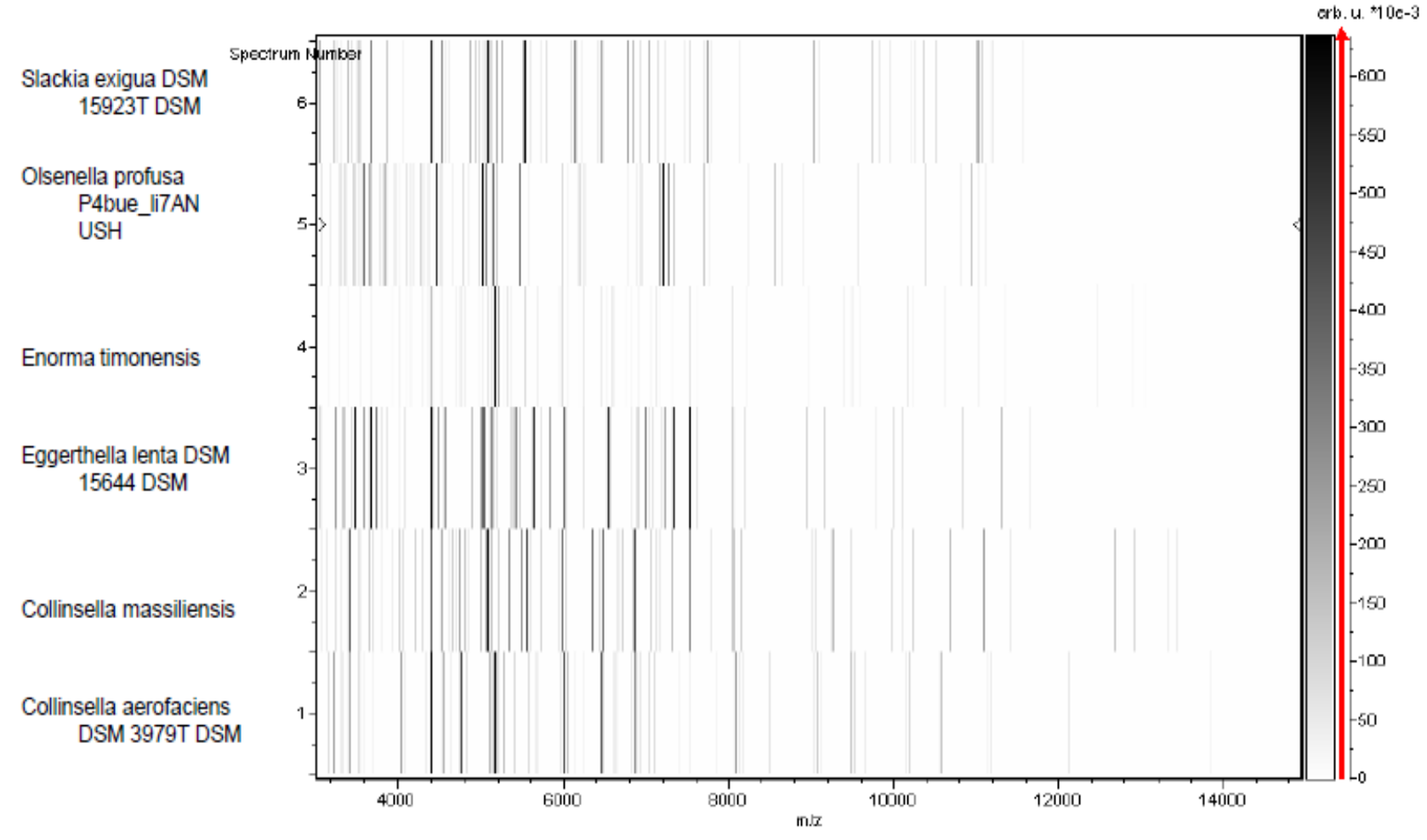

Figure 5: Gel view comparing Collinsella massiliensis strain $\mathrm{GD}^{\top}$ to other members of the family Coriobacteriaceae. The gel view displays the raw spectra of all loaded spectrum files arranged in a pseudo-gel-like look. The $x$-axis records the $\mathrm{m} / \mathrm{z}$ value. The left $\mathrm{y}$-axis displays the running spectrum number originating from subsequent spectra loading. The peak intensity is expressed by a Gray scale scheme code. The color bar and the right $y$-axis indicate the relation between the color a peak is displayed with and the peak intensity in arbitrary units. Displayed species are detailed in the left column.

\section{Genome sequencing information}

\section{Genome project history}

The organism was selected for sequencing on the basis of its phylogenetic position and $16 \mathrm{~S}$ rRNA similarity to members of the genus Colinsella, and is part of a study of the human digestive flora aiming at isolating all bacterial species within human feces [1]. It was the fifth ge- nome of a Colinsella species and the first genome of $C$. massiliensis sp. nov. The GenBank accession number is CAPI00000000 and consists of 15 scaffolds and 118 large contigs. Table 3 shows the project information and its association with MIGS version 2.0 compliance [42].

Table 3: Project information

\begin{tabular}{lll}
\hline MIGS ID & Property & Term \\
\hline MIGS-31 & Finishing quality & High-quality draft \\
MIGS-28 & Libraries used & One 454 paired-end 5-kb library \\
MIGS-29 & Sequencing platforms & 454 GS FLX Titanium \\
MIGS-30 & Fold coverage & $92 \times$ \\
MIGS-32 & Assemblers & Newbler version 2.5 .3 \\
& Gene calling method & Prodigal \\
& INSDC ID /GenBank ID & CAPI00000000 \\
& BioProject ID & PRJEB541 \\
& Genbank Date of Release & $17 / 12 / 2012$ \\
& Project relevance & Study of the human gut microbiome
\end{tabular}


Padmanabhan et al.

Growth conditions and DNA isolation

Collinsella massiliensis strain GD3 ${ }^{\mathrm{T}}$ (= CSUR P902 = DSM 26110) was grown on 5\% sheep bloodenriched Columbia agar (BioMerieux) at $37^{\circ} \mathrm{C}$ in anaerobic atmosphere. Bacteria grown on four Petri dishes were harvested and resuspended in $4 \times 100 \mu \mathrm{L}$ of TE buffer. Then, $200 \mu \mathrm{L}$ of this suspension was diluted in $1 \mathrm{ml}$ TE buffer for lysis treatment that included a 30- minute incubation with $2.5 \mu \mathrm{g} / \mu \mathrm{L}$ lysozyme at $37^{\circ} \mathrm{C}$, followed by an overnight incubation with $20 \mu \mathrm{g} / \mu \mathrm{L}$ proteinase $\mathrm{K}$ at $37^{\circ} \mathrm{C}$. Extracted DNA was then purified using 3 successive phenol-chloroform extractions and ethanol precipitation at $-20^{\circ} \mathrm{C}$ overnight. Following centrifugation, the DNA was resuspended in $52 \mu \mathrm{L}$ TE buffer. The yield and concentration was measured by the Quant-it Picogreen kit (Invitrogen) on the Genios-Tecan fluorometer at $26.3 \mathrm{ng} / \mu \mathrm{l}$.

\section{Genome sequencing and assembly}

Five $\mu \mathrm{g}$ of DNA was mechanically fragmented on Covaris device (KBioScience-LGC Genomics, Teddington, UK) using miniTUBE-red. The DNA fragmentation was visualized through an Agilent 2100 BioAnalyzer on a DNA labchip 7500 with an optimal size of $1.9 \mathrm{~kb}$. A $5 \mathrm{~kb}$ paired-end library was constructed according to the $454 \mathrm{GS}$ FLX Titanium paired-end protocol (Roche). Circularization and nebulization were performed and generated a pattern with an optimal at 567 bp. After PCR amplification through 17 cycles followed by double size selection, the single stranded paired-end library was quantified with the Quant-it Ribogreen kit (Invitrogen) on the Genios Tecan fluorometer at $505 \mathrm{pg} / \mu \mathrm{L}$. The library concentration equivalence was calculated as $8.17 \mathrm{E}+09$ molecules $/ \mu \mathrm{L}$. The library was stored at $-20^{\circ} \mathrm{C}$ until further use.

The paired-end library was clonally amplified with $0.5 \mathrm{cpb}$ and $1 \mathrm{cbp}$ in $4 \mathrm{SV}$-emPCR reactions with the GS Titanium SV emPCR Kit (Lib-L) v2 (Roche). The yields of the emPCR reactions were 9.35 and $14.76 \%$ respectively, in the range of 5 to $20 \%$ from the Roche procedure. The library was loaded on a GS Titanium PicoTiterPlate PTP Kit 70x75 and sequenced with the GS Titanium Sequencing Kit XLR70 (Roche). The run was performed overnight and then analyzed on the cluster through the gsRunBrowser and Newbler assembler (Roche). A total, of 672,867 passed filter wells were obtained and generated $214.2 \mathrm{Mb}$ with a length average of $301 \mathrm{bp}$. These sequences were assembled using Newbler (Roche) with $90 \%$ identity and $40 \mathrm{bp}$ as overlap. The final assembly identified 15 scaffolds and 118 large contigs ( $>1500 \mathrm{bp}$ ) generating a genome size of
$2.32 \mathrm{Mb}$ which corresponds to a coverage of $92 \mathrm{x}$ genome equivalent.

\section{Genome annotation}

Open Reading Frames (ORFs) were predicted using Prodigal [51] with default parameters. However, when predicted ORFs spanned a sequencing gap region, they were excluded. The predicted bacterial protein sequences were searched against the GenBank [52] and Clusters of Orthologous Groups (COG) databases using BLASTP. The tRNAScan-SE [53] and RNAmmer [54] softwares were used to predict tRNAs and rRNAs, respectively. Signal peptides and numbers of transmembrane helices were predicted using SignalP [55] and TMHMM [56], respectively. Mobile genetic elements were predicted using PHAST [57] and RAST [58]. ORFans were identified if their BLASTP $E$-value was lower than $1 \mathrm{e}$ 03 for alignment length greater than 80 amino acids. If alignment lengths were smaller than 80 amino acids, we used an $E$-value of 1e-05. Such parameter thresholds have already been used in previous works to define ORFans. Artemis [59] and DNA Plotter [60] were used for data management and visualization of genomic features, respectively. Mauve alignment tool (version 2.3.1) was used for multiple genomic sequence alignment [61].

To estimate the mean level of nucleotide sequence similarity at the genome level between $C$. massiliensis and the other 4 members of the genus Collinsella (Table 6), we used the Average Genomic Identity of gene Sequences (AGIOS) home-made software [7]. Briefly, this software combines the Proteinortho software [62] for detecting orthologous proteins between genomes compared two by two, then retrieves the corresponding genes and determines the mean percentage of nucleotide sequence identity among orthologous ORFs using the Needleman-Wunsch global alignment algorithm. C. massiliensis strain $\mathrm{GD}^{\mathrm{T}}$ was compared to $C$. intestinalis strain DSM 13280 (GenBank accession number ABHX00000000), C. aerofaciens strain ATCC 25986 (AAVN00000000), C. stercoris strain DSM 13279 (ABXJ00000000), C. tanakaei strain YIT 12063 (ADLS00000000), Eggerthella lenta strain DSM 2243 (CP001726) and Coriobacterium glomerans strain PW2 (CP0002628).

\section{Genome properties}

The genome of $C$. massiliensis strain $\mathrm{GD}^{\mathrm{T}}$ is 2,319,586 bp long (1 chromosome, no plasmid) with a $65.8 \% \mathrm{G}+\mathrm{C}$ content (Table 4 and Figure 6). Of the 2,057 predicted genes, 2,003 were 
protein-coding genes and 54 were RNAs (51 tRNA and 3 rRNA genes). A total of 1,503 genes (73.06\%) were assigned a putative function. A total of 500 genes $(24.30 \%)$ were annotated as hypothetical proteins. The properties and the statistics of the genome are summarized in Tables 4 and 5 . The distribution of genes into COGs functional categories is presented in Table 5. A total of 165 genes were identified as ORFans $(8.02 \%)$.

Table 4: Nucleotide content and gene count levels of the genome

\begin{tabular}{|c|c|c|}
\hline Attribute & Value & $\%$ of total ${ }^{\mathrm{a}}$ \\
\hline Genome size (bp) & $2,319,586$ & \\
\hline DNA G+C content (bp) & $1,526,287$ & 65.8 \\
\hline DNA coding region (bp) & $1,997,199$ & 86.10 \\
\hline Number of replicons & 1 & \\
\hline Extrachromosomal elements & 0 & \\
\hline Total genes & 2,054 & 100 \\
\hline RNA genes & 54 & 2.62 \\
\hline rRNA operons & 1 & \\
\hline Protein-coding genes & 2,003 & 97.37 \\
\hline Genes with function prediction & 1,503 & 73.06 \\
\hline Genes assigned to COGs & 1,370 & 66.60 \\
\hline Genes with peptide signals & 40 & 1.94 \\
\hline Genes with transmembrane helices & 471 & 22.89 \\
\hline CRISPR repeats & 2 & \\
\hline
\end{tabular}

${ }^{a}$ The total is based on either the size of the genome in base pairs or the total number of protein-coding genes in the annotated genome.

Table 5: Number of genes associated with the 25 general COG functional categories

\begin{tabular}{cccl}
\hline Code & Value & \% of total $\mathbf{~}^{\mathbf{a}}$ & \\
\hline J & 135 & 6.73 & Translation \\
A & 0 & 0 & RNA processing and modification \\
K & 113 & 5.64 & Transcription \\
L & 79 & 3.94 & Replication, recombination and repair \\
B & 0 & 0 & Chromatin structure and dynamics \\
D & 18 & 0.89 & Cell cycle control, mitosis and meiosis \\
Y & 0 & 0 & Nuclear structure \\
V & 53 & 2.64 & Defense mechanisms \\
T & 29 & 1.44 & Signal transduction mechanisms \\
M & 81 & 4.04 & Cell wall/membrane biogenesis \\
N & 2 & 0.09 & Cell motility \\
Z & 0 & 0 & Cytoskeleton \\
W & 0 & 0 & Extracellular structures \\
U & 10 & 0.49 & Intracellular trafficking and secretion \\
O & 39 & 1.94 & Posttranslational modification, protein turnover, chaperones \\
C & 69 & 3.44 & Energy production and conversion \\
G & 189 & 9.43 & Carbohydrate transport and metabolism \\
E & 123 & 6.14 & Amino acid transport and metabolism \\
F & 43 & 2.14 & Nucleotide transport and metabolism \\
H & 28 & 1.39 & Coenzyme transport and metabolism \\
I & 28 & 1.39 & Lipid transport and metabolism \\
P & 46 & 2.29 & Inorganic ion transport and metabolism \\
Q & 3 & 0.14 & Secondary metabolites biosynthesis, transport and catabolism \\
R & 165 & 8.23 & General function prediction only \\
S & 117 & 5.84 & Function unknown \\
- & 500 & 24.96 & Not in COGs \\
\hline
\end{tabular}

a The total is based on the total number of protein coding genes in the annotated genome. 


\section{Comparison with other genomes}

The genome of $C$. massiliensis was compared with those of $C$. intestinalis, C. aerofaciens, $C$. stercoris, C. tanakaei, Eggerthella lenta and Coriobacterium glomerans (Table 6). The draft genome of $C$. massiliensis is larger than that of $C$. intestinalis and C. glomerans (2.32, 1.8 and 2.12 $\mathrm{Mb}$, respectively) but smaller than all other other studied genomes (Table 6). In contrast, it exhibits a higher $\mathrm{G}+\mathrm{C}$ content than all other genomes (Table 6). The distribution of genes into COG categories in the genomes from all 5 compared Collinsella species and Coriobacterium glomerans was similar but different from Eggerthella lenta (Figure 7). In addition, $C$. massiliensis shared 867, 947, 953, 1,029, 751 and
841 orthologous genes with C. intestinalis, $C$. aerofaciens, C. stercoris, C. tanakaei, Eggerthella lenta and Coriobacterium glomerans, respectively. Among compared Collinsella genomes except C. massiliensis, AGIOS values ranged from 74.19 between $C$. aerofaciens and $C$. tanakaei to $81.80 \%$ between C. intestinalis and C. stercoris. When C. massiliensis was compared to other Collinsella species, AGIOS values ranged from 74.37 with $C$. tanakaei to $76.52 \%$ with $C$. stercoris (Table 7). In addition, C. massiliensis exhibited AGIOS values of 71.24 and $73.73 \%$ with Eggerthella lenta and Coriobacterium glomerans, respectively (Table 7).

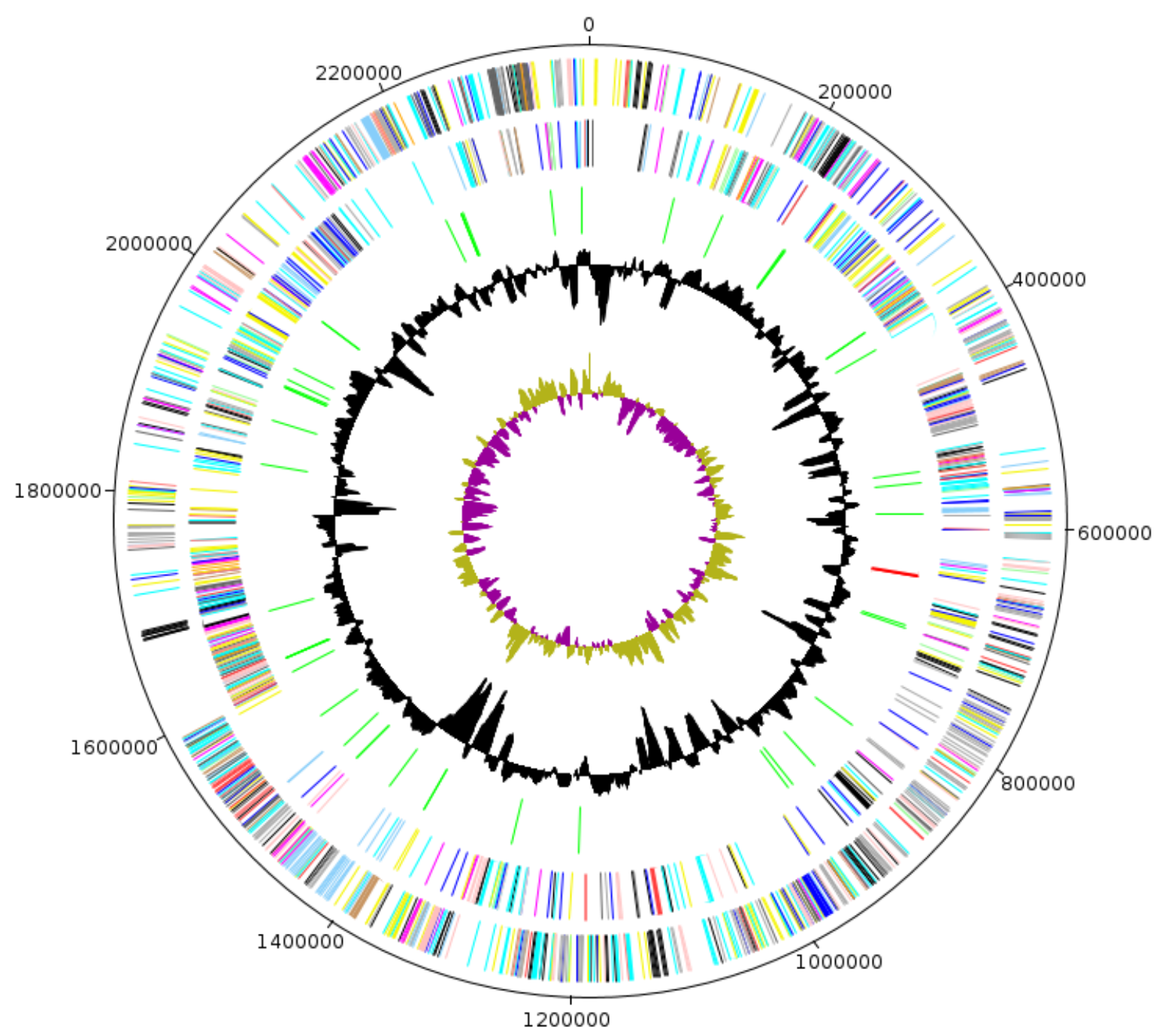

Figure 6: Graphical circular map of the Collinsella massiliensis strain GD3 ${ }^{\top}$ chromosome. From the outside in: open reading frames oriented in the forward (colored by COG categories) direction, open reading frames oriented in the reverse (colored by COG categories) direction, RNA operon (red), and tRNAs (green), GC content plot, and GC skew (purple: negative values, olive: positive values). 


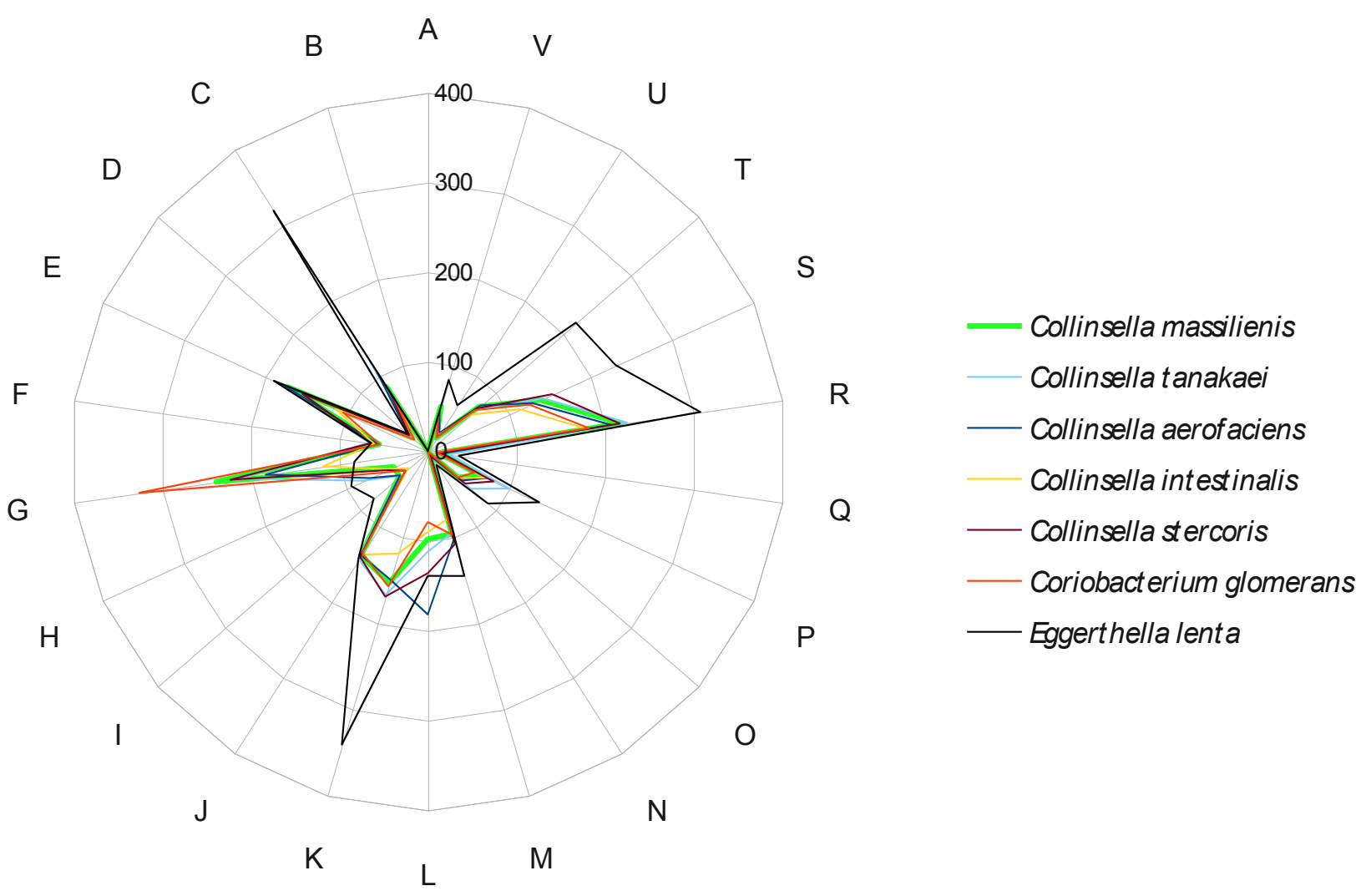

Figure 7: Distribution of functional classes of predicted genes of C. massiliensis sp. nov. strain GD ${ }^{\top}$ (green) and other members of the genus Collinsella, Eggerthella lenta and Coriobacterium glomerans.

Table 6: Genomes used in the genomic comparison and their main characteristics

\begin{tabular}{llrrr}
\hline Organism & GenBank id & Size (Mb) & GC\% & Genes \\
\hline Collinsella massiliensis GD3 & CAPI00000000 & 2.32 & 65.8 & 2,054 \\
Collinsella intestinalis DSM 13280 & ABXH00000000 & 1.8 & 62.5 & 1,846 \\
Collinsella aerofaciens ATCC 25986 & AAVN00000000 & 2.44 & 60.6 & 2,437 \\
Collinsella stercoris DSM 13279 & ABXJ00000000 & 2.4 & 63.2 & 2,585 \\
Collinsella tanakaei YIT 12063 & ADLS00000000 & 2.48 & 60.2 & 2,276 \\
Eggerthella lenta DSM 2243 & CP001726 & 3.63 & 64.2 & 3,184 \\
Coriobacterium glomerans PW2 & CP002628 & 2.12 & 60.4 & 1,858 \\
\hline
\end{tabular}

Table 7: Numbers of orthologous protein-coding genes shared among genomes

\begin{tabular}{lccccccc}
\hline & CM & CA & CI & CS & CT & EL & CG \\
\hline CM & $\mathbf{2 , 0 0 3}$ & 74.61 & 75.95 & 76.51 & 74.37 & 71.24 & 73.73 \\
CA & 947 & $\mathbf{2 , 3 6 7}$ & 74.91 & 75.05 & 74.19 & 68.55 & 71.63 \\
Cl & 867 & 945 & $\mathbf{1 , 7 8 4}$ & 81.80 & 75.67 & 69.28 & 72.42 \\
CS & 953 & 999 & 1,110 & $\mathbf{2 , 5 2 9}$ & 75.70 & 69.88 & 72.53 \\
CT & 1,029 & 1,082 & 1,108 & 1,151 & $\mathbf{2 , 2 1 2}$ & 68.46 & 71.50 \\
EL & 751 & 764 & 740 & 777 & 856 & $\mathbf{3 , 0 7 0}$ & 68.52 \\
CG & 841 & 841 & 818 & 861 & 898 & 639 & $\mathbf{1 , 7 6 8}$ \\
\hline
\end{tabular}

Orthlogues, Lower triangle, AGIOS values upper triangle; number of proteins per genome (bold numbers). $\mathrm{CM}=$ C. massiliensis, $\mathrm{CA}=\mathrm{C}$. aerofaciens, $\mathrm{Cl}=\mathrm{C}$. intestinalis, $\mathrm{CS}=\mathrm{C}$. stercoris, $\mathrm{CT}=\mathrm{C}$. tanakaei, $\mathrm{EL}=$ Eggerthella lenta, $\mathrm{CG}=$ Coriobacterium glomerans. 


\section{Conclusion}

On the basis of phenotypic, phylogenetic and genomic analyses (taxono-genomics), we formally propose the creation of Collinsella massiliensis sp. nov. That contains strain $\mathrm{GD}^{\mathrm{T}}$ as type strain. The strain was isolated from the fecal flora of a 53-year-old woman hospitalized in ICU in Marseille, France, due to a Guillain-Barré syndrome.

\section{Description of Collinsella massiliensis strain sp. nov.}

Collinsella massiliensis (mas.si.li.en'sis. L. masc. adj. massiliensis of Massilia, the Roman name of Marseille, France, where type strain GD3 ${ }^{\mathrm{T}}$ was isolated).

Colonies are grey, translucent and $0.4 \mathrm{~mm}$ in diameter on blood-enriched Columbia agar. Cells are rod shaped with a mean diameter and length of 0.57 and $1.19 \mu \mathrm{m}$, respectively. Optimal growth is achieved anaerobically only. Growth occurs at 37 and $45^{\circ} \mathrm{C}$, with optimal growth observed at $37^{\circ} \mathrm{C}$.

Cells stain Gram-positive, are non-endospore forming and are non-motile. Cells are negative for catalase and oxidase. Positive reactions are observed for acid phosphatase, alkaline phosphatise, naphthol-AS-BI-phosphohydrolase, $\alpha$ galactosidase, $\alpha$-galactosidase, $\alpha$-glucosidase, $\alpha$ fucosidase, leucine arylamidase, proline arylamidase, arginine dihydrolase, serine arylamidase, glycine arylamidase and acidification of D-sorbitol, D-saccharose, xylitol, D-arabitol and potassium-5-ketogluconate. Negative reactions are observed for leucine arylamidase, valine arylamidase, cystin arylamidase, $\beta$-glucuronidase, nitrate reduction, urease, esterase (C4), esterase lipase (C8), lipase (C14), Trypsin, $\alpha$ -

\section{References}

1. Lagier JC, Armougom F, Million M, Hugon P, Pagnier I, Robert C, Bittar F, Fournous G, Gimenez G, Maraninchi M, et al. Microbial culturomics: paradigm shift in the human gut microbiome study. Clin Microbiol Infect 2012; 18:1185-1193. PubMed

2. Genome Online Database.

http://www.genomesonline.org/cgi-bin/GOLD/ index.cgi

3. Tindall BJ, Rosselló-Móra R, Busse HJ, Ludwig W, Kämpfer P. Notes on the characterization of prokaryote strains for taxonomic purposes. Int J Syst Evol Microbiol 2010; 60:249-266. PubMed

4. Stackebrandt E, Ebers J. Taxonomic parameters revisited: tarnished gold standards. Microbiol Today 2006; 33:152-155. chemotrypsin, $\mathrm{N}$-actetyl- $\beta$-glucosaminidase, $\alpha$ mannosidase, $\alpha$-fucosidase, histidin arylamidase, urease, phenylalanine arylamidase, tyrosin arylamidase, leucyl-glycyl arylamidase, alanine arylamidase, arginine arylamidase and fermentation of glycerol, erythritol, D-arabinose, Larabinose, D-ribose, D-xylose, L-xylose, Dadonitol, methyl- $\beta$-D-xylopranoside, D-galactose, D-glucose, D-fructose, D-mannose, L-sorbose, L-rhamnose, dulcitol, inositol, D-mannitol, methyl- $\alpha \mathrm{D}$-xylopranoside, methyl- $\alpha \mathrm{D}$-glucopranoside, $\mathrm{N}$-acetylglucosamine, amygdalin, arbutin, aesculin ferric citrate, salicin, Dcellobiose, D-maltose, D-lactose, D-mellibiose, Dtrehalose, inulin, D-melezitose, D-raffinose, amidon, glycogen, gentiobiose, D-turanose, Dlyxose, D-tagatose, L-fucose, L-arabitol, potassium gluconate and potassium 2-ketogluconate. Positive reactions were recorded for acid phosphatase, naphthol-AS-BI-phosphohydrolase, $\alpha$ galactosid-ase, alkaline phosphatase, leucine arylamidase, valine arylamidase, a-glucosidatse and $\beta$ glucosidasee. Cells are susceptible to penicillin G, amoxicillin, amoxicillin-clavulanic acid, ceftriaxone, imipenem, metronidazole, vancomycin, rifampicin but resistant to erythromycin, gentamicin, ciprofloxacin and trimethoprim/sulfa-methoxazole.

The 16S rRNA and genome sequences are deposited in GenBank and EMBL under accession numbers JX424766 and CAPI00000000, respectively. The $\mathrm{G}+\mathrm{C}$ content of the genome is $65.8 \%$. The habitat of the microorganism is the human digestive tract. The type strain GD3 $^{\mathrm{T}}(=\mathrm{CSUR}$ P902 = DSM 26110) was isolated from the fecal flora of a French Caucasoid woman who suffered from Guillain-Barré syndrome. This strain was isolated in Marseille, France.

5. Wayne LG, Brenner DJ, Colwell PR, Grimont PAD, Kandler O, Krichevsky MI, Moore LH, Moore WEC, Murray RGE, Stackebrandt E, et al. Report of the ad hoc committee on reconciliation of approaches to bacterial systematic. Int J Syst Bacteriol 1987; 37:463-464.

6. Rossello-Mora R. DNA-DNA Reassociation Methods Applied to Microbial Taxonomy and Their Critical Evaluation. In: Stackebrandt E (ed), Molecular Identification, Systematics, and population Structure of Prokaryotes. Springer, Berlin, 2006; p. 23-50.

7. Ramasamy D, Mishra AK, Lagier JC, Padhmanabhan R, Rossi-Tamisier M, Sentausa E, Raoult D, Fournier PE. A polyphasic strategy incorporating genomic data for the taxonomic 
description of new bacterial species. Int J Syst

Evol Microbiol 2014; (In press). PubMed

8. Kokcha S, Mishra AK, Lagier JC, Million M, Leroy Q, Raoult D, Fournier PE. Noncontiguous finished genome sequence and description of Bacillus timonensis sp. nov. Stand Genomic Sci 2012; 6:346-355. PubMed

9. Lagier JC, El Karkouri K, Nguyen TT, Armougom F, Raoult D, Fournier PE. Non-contiguous finished genome sequence and description of Anaerococcus senegalensis sp. nov. Stand Genomic Sci 2012; 6:116-125. PubMed

10. Mishra AK, Gimenez G, Lagier JC, Robert C, Raoult D, Fournier PE. Non-contiguous finished genome sequence and description of Alistipes senegalensis sp. nov. Stand Genomic Sci 2012; 6:304-314.

11. Lagier JC, Armougom F, Mishra AK, Ngyuen TT, Raoult D, Fournier PE. Non-contiguous finished genome sequence and description of Alistipes timonensis sp. nov. Stand Genomic Sci 2012; 6:315-324. PubMed

12. Mishra AK, Lagier JC, Robert C, Raoult D, Fournier PE. Non-contiguous finished genome sequence and description of Clostridium senegalense sp. nov. Stand Genomic Sci 2012; 6:386-395. PubMed

13. Mishra AK, Lagier JC, Robert C, Raoult D, Fournier PE. Non-contiguous finished genome sequence and description of Peptoniphilus timonensis sp. nov. Stand Genomic Sci 2012; 7:1-11. PubMed

14. Mishra AK, Lagier JC, Rivet R, Raoult D, Fournier PE. Non-contiguous finished genome sequence and description of Paenibacillus senegalensis sp. nov. Stand Genomic Sci 2012; 7:70-81. PubMed

15. Lagier JC, Gimenez G, Robert C, Raoult D, Fournier PE. Non-contiguous finished genome sequence and description of Herbaspirillum massiliense sp. nov. Stand Genomic Sci 2012; 7:200-209. PubMed

16. Kokcha S, Ramasamy D, Lagier JC, Robert C, Raoult D, Fournier PE. Non-contiguous finished genome sequence and description of Brevibacterium senegalense sp. nov. Stand Genomic Sci 2012; 7:233-245. PubMed

17. Ramasamy D, Kokcha S, Lagier JC, N'Guyen TT, Raoult D, Fournier PE. Non-contiguous finished genome sequence and description of Aeromicrobium massilense sp. nov. Stand Genomic Sci 2012; 7:246-257. PubMed

18. Lagier JC, Ramasamy D, Rivet R, Raoult D, Fournier PE. Non-contiguous finished genome sequence and description of Cellulomonas massiliensis sp. nov. Stand Genomic Sci 2012; 7:258-270. $\underline{\text { PubMed }}$

19. Lagier JC, Karkouri K, Rivet R, Couderc C, Raoult D, Fournier PE. Non contiguous-finished genome sequence and description of Senegalemassilia anaerobia gen. nov., sp. nov. Stand Genomic Sci 2013; 7:343-356. PubMed

20. Mishra AK, Hugon $P$, Nguyen TT, Robert C, Couderc C, Raoult D, Fournier PE. Non contiguous-finished genome sequence and description of Peptoniphilus obesi sp. nov. Stand Genomic Sci 2013; 7:357-369. PubMed

21. Mishra AK, Lagier JC, Nguyen TT, Raoult D, Fournier PE. Non contiguous-finished genome sequence and description of Peptoniphilus senegalensis sp. nov. Stand Genomic Sci 2013; 7:357-369. PubMed

22. Lagier JC, Karkouri K, Mishra AK, Robert C, Raoult D, Fournier PE. Non contiguous-finished genome sequence and description of Enterobacter massiliensis sp. nov. Stand Genomic Sci 2013; 7:399-412. PubMed

23. Hugon P, Ramasamy D, Rivet R, Raoult D, Fournier PE. Non contiguous-finished genome sequence and description of Alistipes obesi sp. nov. Stand Genomic Sci 2013; 7:427-439. PubMed

24. Hugon P, Mishra AK, Nguyen TT, Raoult D, Fournier PE. Non-contiguous finished genome sequence and description of Brevibacillus massiliensis sp. nov. Stand Genomic Sci 2013; 8:1-14. PubMed

25. Mishra AK, Hugon P, Nguyen TT, Raoult D, Fournier PE. Non contiguous-finished genome sequence and description of Enorma massiliensis gen. nov., sp. nov., a new member of the Family Coriobacteriaceae. Stand Genomic Sci 2013; 8:290-305. PubMed

26. Ramasamy D, Lagier JC, Gorlas A, Raoult D, Fournier PE. Non contiguous-finished genome sequence and description of Bacillus massiliosenegalensis sp. nov. Stand Genomic Sci 2013; 8:264-278. PubMed

27. Ramasamy D, Lagier JC, Nguyen TT, Raoult D, Fournier PE. Non contiguous-finished genome sequence and description of Dielma fastidiosa gen. nov., sp. nov., a new member of the Family Erysipelotrichaceae. Stand Genomic Sci 2013; 8:336-351. PubMed

28. Mishra AK, Pfleiderer A, Lagier JC, Robert C, Raoult D, Fournier PE. Non contiguous-finished genome sequence and description of Bacillus massilioanorexius sp. nov. Stand Genomic Sci 2013; 8:465-479. PubMed 
Padmanabhan et al.

29. Hugon P, Ramasamy D, Robert C, Couderc C, Raoult D, Fournier PE. Non-contiguous finished genome sequence and description of Kallipyga massiliensis gen. nov., sp. nov., a new member of the family Clostridiales Incertae Sedis XI. Stand Genomic Sci 2013; 8:500-515. PubMed

30. Padhmanabhan R, Lagier JC, Dangui NPM, Michelle C, Couderc C, Raoult D, Fournier PE. Non-contiguous finished genome sequence and description of Megasphaera massiliensis. Stand Genomic Sci 2013; 8:525-538. PubMed

31. Mishra AK, Edouard S, Dangui NPM, Lagier JC, Caputo A, Blanc-Tailleur C, Ravaux I, Raoult D, Fournier PE. Non-contiguous finished genome sequence and description of Nosocomiicoccus massiliensis sp. nov. Stand Genomic Sci 2013; 9:205-219. PubMed

32. Mishra AK1, Lagier JC, Robert C, Raoult D, Fournier PE. Genome sequence and description of Timonella senegalensis gen. nov., sp. nov., a new member of the suborder Micrococcineae. Stand Genomic Sci 2013; 8:318-335. PubMed

33. Keita MB, Diene SM, Robert C, Raoult D, Fournier PE. Non contiguous-finished genome sequence and description of Bacillus massiliogorillae sp. nov. Stand Genomic Sci 2013; 9:93-105. PubMed

34. Mediannikov O, El Karkouri K, Robert C, Fournier PE, Raoult D. Non contiguous-finished genome sequence and description of Bartonella florenciae sp. nov. Stand Genomic Sci 2013; 9:185-196. PubMed

35. Lo Cl, Mishra AK, Padhmanabhan R, Samb Ba B, Gassama Sow A, Robert C, Couderc C, Faye $\mathrm{N}$, Raoult D, Fournier PE, Fenollar F. Non contiguous-finished genome sequence and description of Clostridium dakarense sp. nov. Stand Genomic Sci 2013; 9:14-27. PubMed

36. Mishra AK, Hugon $P$, Robert C, Raoult D, Fournier PE. Non contiguous-finished genome sequence and description of Peptoniphilus grossensis sp. nov. Stand Genomic Sci 2012; 7:320-330. PubMed

37. Mediannikov O, El Karkouri K, Diatta G, Robert C, Fournier PE, Raoult D. Non contiguousfinished genome sequence and description of Bartonella senegalensis sp. nov. Stand Genomic Sci 2013; 8:279-289. $\underline{\text { PubMed }}$

38. Mishra AK, Lagier JC, Pfleiderer A, Nguyen TT, Caputo A, Raoult D, Fournier PE. Non contiguous-finished genome sequence and description of Holdemania massiliensis sp. nov. Stand Genomic Sci 2013; 9:395-409.

39. Kageyama A, Benno Y, Nakase T. Phylogenetic and phenotypic evidence for the transfer ofEubacterium aerofaciens to the genus
Collinsella as Collinsella aerofaciens gen. nov., comb. nov. Int / Syst Bacteriol 1999; 49:557565. PubMed

40. Kageyama A, Benno Y. Emendation of genus Collinsella and proposal of Collinsella stercoris sp. nov. and Collinsella intestinalis sp. nov. Int J Syst Evol Microbiol 2000; 50:1767-1774. $\underline{\text { PubMed }}$

41. Nagai F, Watanabe Y, Morotomi M. Slackia piriformis sp. nov. and Collinsella tanakaei sp. nov., new members of the family Coriobacteriaceae, isolated from human faeces. Int J Syst Evol Microbiol 2010; 60:2639-2646. PubMed

42. Field D, Garrity G, Gray T, Morrison N, Selengut J, Sterk P, Tatusova T, Thomson N, Allen MJ, Angiuoli SV, et al. The minimum information about a genome sequence (MIGS) specification. Nat Biotechnol 2008; 26:541-547. PubMed

43. Woese CR, Kandler O, Wheelis ML. Towards a natural system of organisms: proposal for the domains Archaea, Bacteria, and Eucarya. Proc Natl Acad Sci USA 1990; 87:4576-4579. PubMed

44. Garrity GM, Holt JG. The Road Map to the Manual. In: Garrity GM, Boone DR, Castenholz RW (eds), Bergey's Manual of Systematic Bacteriology, Second Edition, Volume 1. Springer, New York 2001; 119-169.

45. Stackebrandt E, Rainey FA, Ward-Rainey NL. Proposal for a new hierarchic classification system, Actinobacteria classis nov. Int I Syst Bacteriol 1997; 47:479-491.

46. Zhi XY, Li WJ, Stackebrandt E. An update of the structure and 16S rRNA gene sequence-based definition of higher ranks of the class Actinobacteria, with the proposal of two new suborders and four new families and emended descriptions of the existing higher taxa. Int I Syst Evol Microbiol 2009; 59:589-608. PubMed

47. Wade WG, Downes J, Dymock D, Hiom S, Weightman AJ, Dewhirst FE, Paster BJ, Tzellas $\mathrm{N}$, Coleman B. The family Coriobacteriaceae: reclassification of Eubacterium exiguum (Poco et al. 1996) and Peptostreptococcus heliotrinreducens(Lanigan 1976) as Slackia exigua gen. nov., comb. nov. and Slackia heliotrinireducens gen. nov., comb. nov., and Eubacterium lentum (Prevot 1938) as Eggerthella lenta gen. nov., comb. nov. Int J Syst Bacteriol 1999; 49:595-600. PubMed

48. Ashburner M, Ball CA, Blake JA, Botstein D, Butler H, Cherry JM, Davis AP, Dolinski K, Dwight SS, Eppig JT, et al. Gene ontology: tool for the unification of biology. The Gene Ontol- 
ogy Consortium. Nat Genet 2000; 25:25-29. PubMed

49. 16S Yourself database. (http://www.mediterranee-infection.com/article .php?larub=152\&titre=16s-yourself).

50. Seng P, Drancourt M, Gouriet F, La SB, Fournier $\mathrm{PE}$, Rolain JM, et al. Ongoing revolution in bacteriology: routine identification of bacteria by matrix-assisted laser desorption ionization timeof-flight mass spectrometry. Clin Infect Dis 2009; 49:543-551. PubMed

51. Prodigal. http://prodigal.ornl.gov

52. Benson DA, Karsch-Mizrachi I, Clark K, Lipman DJ, Ostell J, Sayers EW. GenBank. Nucleic Acids Res 2012; 40:D48-D53. PubMed

53. Lowe TM, Eddy SR. tRNAscan-SE: a program for improved detection of transfer RNA genes in genomic sequence. Nucleic Acids Res 1997; 25:955-964. PubMed

54. Lagesen K, Hallin P, Rodland EA, Staerfeldt HH, Rognes T, Ussery DW. RNAmmer: consistent and rapid annotation of ribosomal RNA genes. Nucleic Acids Res 2007; 35:3100-3108. PubMed

55. Bendtsen JD, Nielsen H, von Heijne G, Brunak S. Improved prediction of signal peptides: SignalP 3.0. J Mol Biol 2004; 340:783-795. PubMed

56. Krogh A, Larsson B, von Heijne G, Sonnhammer EL. Predicting transmembrane protein to- pology with a hidden Markov model: application to complete genomes. J Mol Biol 2001; 305:567-580. PubMed

57. Zhou Y, Liang Y, Lynch KH, Dennis JJ, Wishart DS. PHAST: a fast phage search tool. Nucleic Acids Res 2011; 39:W347-W352. PubMed

58. Aziz RK, Bartels D, Best AA, Dejongh M, Disz T, Edwards RA, Formsma K, Gerdes S, Glass EM, Kubal M, et al. The RAST Server: rapid annotations using subsystems technology. BMC Genomics 2008; 9:75. PubMed

59. Rutherford K, Parkhill J, Crook J, Horsnell T, Rice P, Rajandream MA, Barrell B. Artemis: sequence visualization and annotation. Bioinformatics 2000; 16:944-945. PubMed

60. Carver T, Thomson N, Bleasby A, Berriman M, Parkhill J. DNAPlotter: circular and linear interactive genome visualization. Bioinformatics 2009; 25:119-120. PubMed

61. Darling AC, Mau B, Blattner FR, Perna NT. Mauve: multiple alignment of conserved genomic sequence with rearrangements. Genome Res 2004; 14:1394-1403. PubMed

62. Lechner M, Findeib S, Steiner L, Marz M, Stadler PF, Prohaska SJ. Proteinortho: Detection of (Co-)orthologs in large-scale analysis. BMC Bioinformatics 2011; 12:124. PubMed 